\title{
Opposite regulation of epithelial-to-mesenchymal transition and cell invasiveness by periostin between prostate and bladder cancer cells
}

\author{
CHUL JANG KIM ${ }^{1}$, KANAMI SAKAMOTO ${ }^{2,3}$, YUKIHIRO TAMBE ${ }^{2}$ and HIROKAZU INOUE ${ }^{2}$ \\ ${ }^{1}$ Department of Urology, Kohka Public Hospital, 3-39 Rokushin, Kohka, Shiga 528-0014; ${ }^{2}$ Division \\ of Microbiology and Infectious Diseases, Department of Pathology, Shiga University of Medical \\ Science, Setatsukinowa-cho, Otsu, Shiga 520-2192; ${ }^{3}$ Department of Cell Biology and Bioscience, \\ The Nagahama Institute of Bioscience and Technology, Nagahama, Shiga 526-0829, Japan
}

Received December 22, 2010; Accepted February 23, 2011

DOI: 10.3892/ijo.2011.997

\begin{abstract}
We previously showed that periostin expression is downregulated in human bladder cancer tissues and that ectopic expression of periostin suppresses the invasiveness of bladder cancer cells. However, in most other human cancers studied, the expression of periostin promotes cell invasiveness. In the present study, we investigated the regulation of the epithelialto-mesenchymal transition (EMT) and cell invasiveness by periostin in bladder and prostate cancer cell lines, and found opposite regulation of EMT and cell invasiveness by periostin. Periostin upregulated E-cadherin expression in bladder cancer cells but downregulated it in prostate cancer cells. Periostin suppressed cell invasiveness in bladder cancer cells but promoted it in prostate cancer cells. Snail, a negative regulator of E-cadherin, was upregulated by periostin in prostate cancer cells, while Twist, another negative regulator of E-cadherin, was downregulated in bladder cancer cells. The C-terminal region of periostin was sufficient for these functions in bladder cancer cells but not in prostate cancer cells. Knockdown of endogenous Snail by siRNA suppressed cell invasiveness in prostate cancer cells expressing periostin. Periostin also suppressed Akt phosphorylation in bladder cancer cells but enhanced it in prostate cancer cells. Treatment with Akt inhibitor increased E-cadherin expression and suppressed both Twist expression and cell invasiveness of bladder cancer cells. These results indicate that Akt signaling plays a role in the cell-type-dependent regulation of E-cadherin expression and cell invasiveness by periostin via Snail and Twist.
\end{abstract}

Correspondence to: Dr Hirokazu Inoue, Division of Microbiology and Infectious Diseases, Department of Pathology, Shiga University of Medical Science, Setatsukinowa-cho, Otsu, Shiga 520-2192, Japan E-mail: hirokazu@belle.shiga-med.ac.jp

Key words: periostin, cell invasiveness, epithelial-to-mesenchymal transition, prostate cancer, bladder cancer

\section{Introduction}

Local invasion can be considered an initial and essential step in the malignancy of carcinomas, leading to the generation of usually fatal distant metastasis. Tumor invasion appears to be controlled by a coordinated series of cellular and molecular processes that enable tumor cells to dissociate and migrate from the primary tumor. The changes in cell adhesion and migration during tumor invasion are reminiscent of an important developmental process called the epithelial-to-mesenchymal transition (EMT) $(1,2)$. One of the hallmarks of EMT is the functional loss of E-cadherin, which is currently thought to be a suppressor of invasion during carcinoma progression (3). The characterization of E-cadherin regulation during malignant progression has provided important insights into the molecular mechanisms involved in tumor invasion.

Periostin has been shown to facilitate the migration and differentiation of cells that have undergone EMT, both during embryogenesis and under pathological conditions (4). Various aggressive tumors, including colon cancer (5), breast cancer (6), lung cancer (7), head and neck cancer (8), oral cancer (9), and pancreatic cancer (10), are characterized by overexpression of periostin. High expression of periostin is noted during EMT of cancer cells in non-small cell lung cancer (11). These reports have suggested that periostin is involved in tumor spread, invasion and metastasis. On the other hand, we have reported that the downregulation of periostin mRNA expression is associated with higher grade of human bladder cancers, and that ectopic expression of periostin by a retroviral vector could suppress the in vitro invasiveness of human bladder cancer cells and the in vivo lung metastasis of mouse melanoma cells without affecting cell proliferation or tumor growth in nude mice (12). Our mutational analysis revealed that the C-terminal region of periostin, where alternative splicing events occur, is necessary and sufficient for the suppression of both the in vitro cell invasiveness and the in vivo lung metastasis (12). We have also demonstrated that the loss of wild-type (WT) periostin by downregulation and/or alternative splicing in the C-terminal region is closely correlated with the development of bladder cancer (13). In the present study, to clarify the molecular basis 
of this discrepancy in the function of periostin on the invasiveness between human bladder cancer cells and other cancer cells, we investigated the activities of periostin to regulate the in vitro invasiveness and EMT-related molecules in prostate and bladder cancer cell lines, and found that periostin regulates E-cadherin expression and the cell invasiveness in a cell-type dependent manner via Akt phosphorylation and negative regulators of E-cadherin transcription, Snail and Twist.

\section{Materials and methods}

Cell lines. Human bladder cancer cell lines (SBT31A and T24), prostate cancer cell lines (PC3 and DU145) and an embryonic kidney cell line, 293T, were cultured in RPMI-1640 (PC3, DU145 and SBT31A) and DMEM (T24 and 293T) medium supplemented with $10 \%$ fetal calf serum (FCS), penicillin $(100 \mathrm{U} / \mathrm{ml})$ and streptomycin $(100 \mu \mathrm{g} / \mathrm{ml})$ at $37^{\circ} \mathrm{C}$ in a humidified $5 \% \mathrm{CO}_{2}$ atmosphere. The $293 \mathrm{~T}$ cell line was used for the preparation of recombinant retroviruses.

Plasmids and transfection. The expression plasmid of human periostin was described previously (13). The expression plasmids of WT and the C-terminal region (C-term) mutant of periostin were also described previously $(12,14)$. All plasmids were prepared using Qiagen columns (Qiagen, Hilden, Germany). Plasmids were transfected in 60-mm dishes using LipofectaminePlus reagent (Invitrogen, Carlsbad, CA) according to the manufacturer's protocol.

Preparation of recombinant retrovirus and virus infection. The pCXbsr retrovirus vectors carrying WT or C-term mutant periostin cDNA $(12,13)$ were transfected into $293 \mathrm{~T}$ cells with the helper plasmid pCL-ampho (15) using Lipofectamine-Plus reagent. The amphotropic retroviruses in the culture medium were collected $48 \mathrm{~h}$ after transfection, filtered and stored at $-80^{\circ} \mathrm{C}$ until use. For viral infection, $2 \times 10^{5}$ human cancer cells were plated onto $60-\mathrm{mm}$ dishes and cultured overnight at $37^{\circ} \mathrm{C}$. After polybrene treatment $(2 \mu \mathrm{g} / \mathrm{ml})$ for $30 \mathrm{~min}$, the retroviruses were added to the cultures, which were then incubated for $1 \mathrm{~h}$ at $37^{\circ} \mathrm{C}$. After 7 days of incubation in selection medium containing $5 \mu \mathrm{g} / \mathrm{ml}$ of blasticidin-S (Invitrogen), the blasticidin-resistant colonies were pooled and used for assays.

In vitro invasion assay. The in vitro invasive potential of the cancer cells was determined using Matrigel ${ }^{\mathrm{TM}}$ Basement Membrane Matrix Invasion Chambers (chamber size, $6.4 \mathrm{~mm}$; membrane surface area, $0.3 \mathrm{~cm}^{2}$; pore size, $8 \mu \mathrm{m}$; BD Biosciences, Bedford, MA) following the manufacturer's instructions. Briefly, the culture supernatant used as a chemoattractant was prepared by culturing NIH3T3 cells in DMEM supplemented with $0.1 \%$ bovine albumin, $0.005 \% \mathrm{~L}(+)$ ascorbic acid, penicillin $(100 \mathrm{U} / \mathrm{ml})$ and streptomycin $(100 \mu \mathrm{g} / \mathrm{ml})$ at $37^{\circ} \mathrm{C}$ for $24 \mathrm{~h}$. Then, $750 \mu \mathrm{l}$ of the culture supernatant was added to the plate well as a chemoattractant, and $500 \mu \mathrm{l}$ of cell suspension at $1 \times 10^{5}$ SBT31A cells $/ \mathrm{ml}, 2 \times 10^{4} \mathrm{~T} 24$ cells $/ \mathrm{ml}, 2 \times 10^{4} \mathrm{PC} 3 \mathrm{cells} / \mathrm{ml}$ or $3 \times 10^{4}$ DU145 cells $/ \mathrm{ml}$ was added to each chamber. The chambers were incubated for 4 (SBT31A), 3 (PC3 and DU145) or 2 days (T24) in a humidified $5 \% \mathrm{CO}_{2}$ atmosphere. Noninvasive cells were removed from the upper surface of the membrane with a cotton swab. The invasive cells on the under- side of the membrane were stained with Diff-Quik ${ }^{\mathrm{TM}}$ stain (Kokusaishiyaku, Kobe, Japan) and counted under a microscope.

Antibodies. Anti-periostin (ab14041, rabbit polyclonal) antibody was purchased from Abcam (Tokyo, Japan). E-cadherin (mouse monoclonal) antibody was purchased from BD Biosciences. Anti-vimentin (mouse monoclonal), anti-Snail (C15D3, rabbit monoclonal), anti-Akt (rabbit polyclonal), and anti-phospho Akt (Ser473, mouse monoclonal) antibodies were purchased from Cell Signaling Technology (Danvers, MA). Anti-Twist (H81, rabbit polyclonal) antibody was purchased from Santa Cruz Biotechnology (Santa Cruz, CA). Anti- $\alpha$-tubulin (T9026, mouse monoclonal) antibody was purchased from SigmaAldrich (St. Louis, MO).

Immunoblotting. Cells were lysed in Laemmli-SDS buffer, subjected to SDS-polyacrylamide gel electrophoresis, and electro-transferred to membrane filters (Immobilon-P, Millipore Corp., Billerica, MA). The filters were incubated with a primary antibody in TBS-T (10 mM Tris-HCl, $\mathrm{pH} 7.6$, $150 \mathrm{mM}$ sodium chloride, $0.1 \%$ Tween-20) containing $2 \%$ bovine serum albumin (BSA) overnight and incubated for $1 \mathrm{~h}$ in horseradish peroxidase-conjugated anti-mouse or antirabbit IgG (GE Healthcare, Buckinghamshire, UK) diluted 1:10,000 in TBS-T containing $2 \%$ BSA. Immuno-reactivity was detected by the ECL system (GE Healthcare).

Reverse transcriptase-polymerase chain reaction ( $R T-P C R)$ analysis. Total RNA was isolated from cultured cells using TRIzol reagent (Invitrogen). Isolated RNA was used for firststrand cDNA synthesis with Superscript II (Invitrogen) and an oligo-dT primer for $50 \mathrm{~min}$ at $42^{\circ} \mathrm{C}$. The cDNA was PCRamplified by Taq DNA polymerase (Takara, Otsu, Japan) with primers specific for E-cadherin Type I, 5'-CGACCCAACCC AAGAATCTA-3' (forward) and 5'-AGGCTGTGCCTTCCT ACAGA-3' (reverse); E-cadherin Type II, 5'-GCCAGGTATGA GATCGTGGT-3' (forward) and 5'-GTGTCTTCAGGCACG ACAAA-3' (reverse) (16). PCR reaction was performed with 30 cycles consisting of denaturation at $94^{\circ} \mathrm{C}$ for $30 \mathrm{sec}$, annealing at $55^{\circ} \mathrm{C}$ for $30 \mathrm{sec}$, and extension at $72^{\circ} \mathrm{C}$ for $1 \mathrm{~min}$, followed by a final extension for $7 \mathrm{~min}$. The glyceraldehyde-3-phosphate dehydrogenase (GAPDH) primers were GAPDH-5F (5'-ACCA CAGTCCATGCCATCAC-3') and GAPDH-3R (5'-TCCACC ACCCTGTTGCTGTA-3'). Thirty cycles of PCR for GAPDH were performed with annealing at $60^{\circ} \mathrm{C}$. The PCR products were separated by electrophoresis in a $1.5 \%$ agarose gel containing ethidium bromide and then photographed.

In vitro cell proliferation. Growth curves were determined by culturing the cancer cells in $35-\mathrm{mm}$ culture plates. Briefly, cells were suspended in complete cell culture medium and plated at $2 \times 10^{4}$ cells in each culture plate, and the cultures in triplicate were kept at $37^{\circ} \mathrm{C}$ in a humidified atmosphere containing $5 \% \mathrm{CO}_{2}$ in air. Viable cells were counted using a hemocytometer.

Pharmacological treatments. 1L-6-hydroxymethyl-chiroinositol 2(R)-2-O-methyl-3-O-octadecylcarbonate (Akt inhibitor) (Calbiochem, San Diego, CA) was prepared in DMSO and stored 


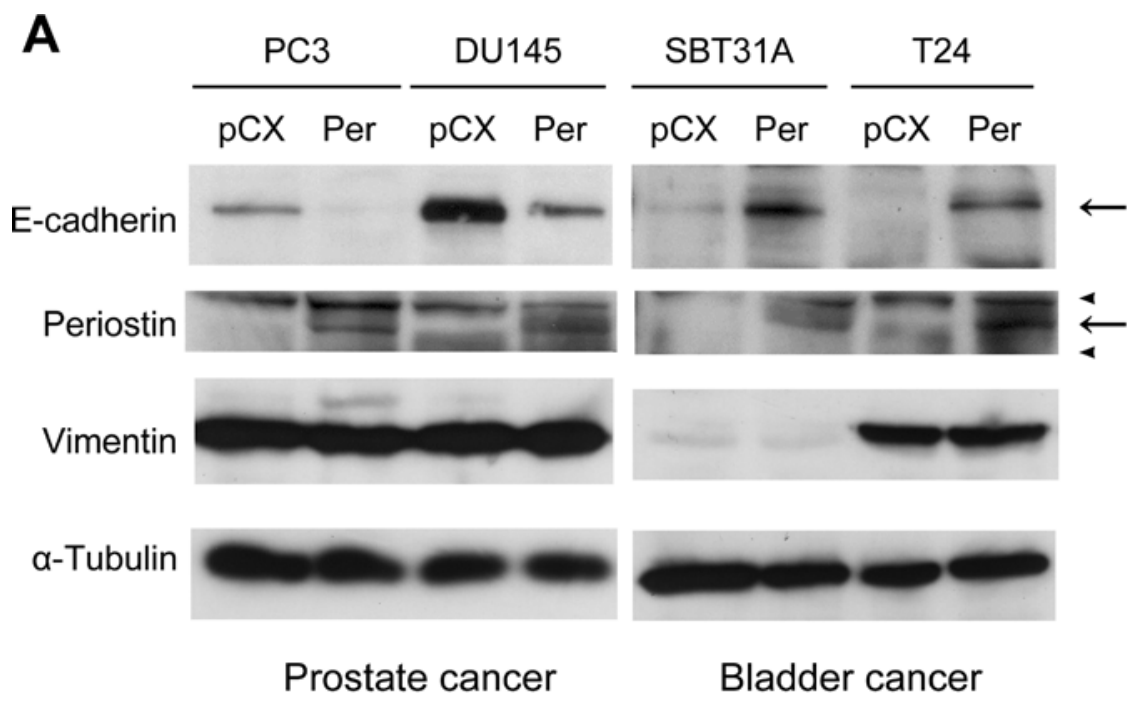

B

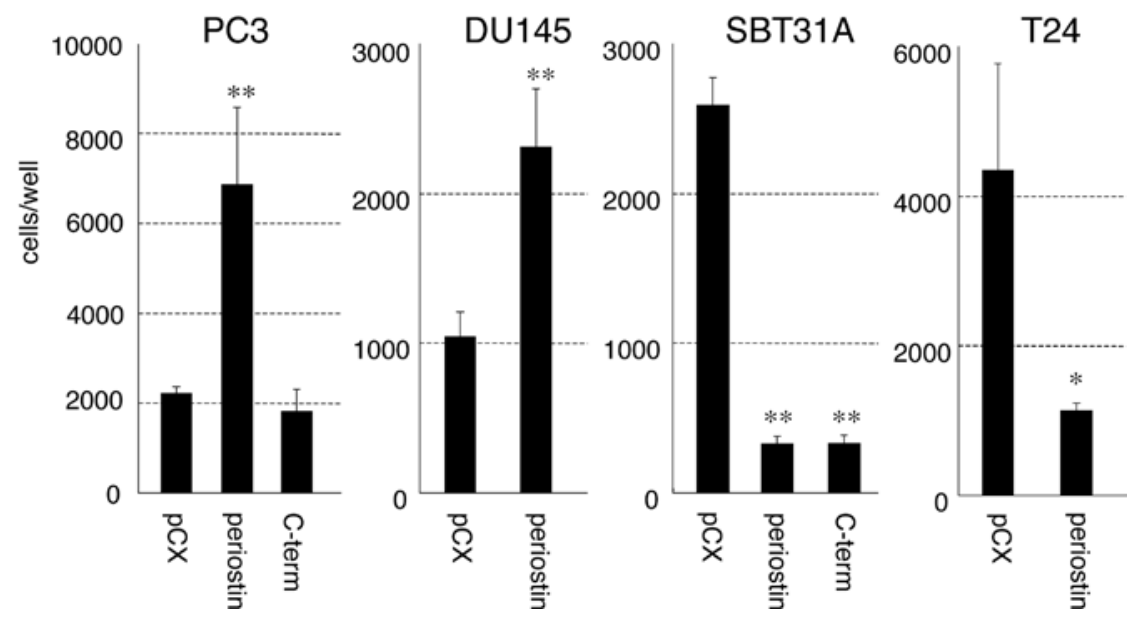

Figure 1. Effects of periostin expression on EMT and invasiveness of prostate and bladder cancer cell lines. (A) Alterations of the expression of E-cadherin and vimentin by periostin in prostate (PC3 and DU145) and bladder (SBT31A and T24) cancer cell lines. Cell lysate was separated by SDS-PAGE and proteins were detected by immunoblotting with the anti-E-cadherin, anti-periostin and anti-vimentin antibodies. The expression of $\alpha$-tubulin was used as an internal control. The arrows show the bands of E-cadherin and periostin. The arrowheads show non-specific bands. (B) Alterations of the cell invasiveness by wild-type periostin (periostin) and the C-terminal region of periostin (C-term) in PC3, DU145, SBT31A and T24 cells. The numbers of cells invading through the Matrigel are shown. Each sample was assayed in triplicate. Bars, \pm SD of triplicate chambers for each experiment. ${ }^{*} \mathrm{p}<0.001,{ }^{* *} \mathrm{p}<0.01$, as compared to the control cell line (pCX).

at $-20^{\circ} \mathrm{C}$. Cancer cells were plated at $1 \times 10^{6}$ cells $/ 60-\mathrm{mm}$ dish in RPMI-1640 medium containing $10 \%$ FCS and incubated for $24 \mathrm{~h}$. The medium was then changed to RPMI-1640 medium with $0.1 \%$ FCS, and the cells were incubated overnight. The cells were then treated with Akt inhibitor $(25 \mu \mathrm{M})$ for $24 \mathrm{~h}$. In all experiments, the same volume of DMSO was added to control samples.

Small interfering RNAs (siRNA). The siRNAs targeting human Snaill (HSS143995) and scramble negative control RNA duplexes were purchased from Invitrogen. The cells were transfected with RNA duplexes using Lipofectamine 2000 reagents (Invitrogen) following the manufacturer's protocol.

Statistical analysis. Student's t-test was used for statistical analysis of in vitro invasion and cell proliferation assays. Only variables with $\mathrm{p}<0.05$ were considered significant.

\section{Results}

Effect of periostin on in vitro invasiveness of cancer cells. To clarify the molecular basis of the discrepancy in the function of periostin on invasiveness between human bladder cancer cells and other cancer cells, we compared the activities of periostin in the regulation of the in vitro invasiveness and EMT in prostate and bladder cancer cell lines under the same experimental conditions. An amphotropic retrovirus expressing the periostin cDNA ( $\mathrm{pCXbsr} /$ Per) and the control vector retrovirus (pCXbsr) were introduced into two prostate (PC3 and DU145) and two bladder (SBT31A and T24) cancer cell lines. Infected cells were selected in medium containing blasticidin. Immunoblot analysis with anti-periostin antibody showed that PC3, DU145, SBT31A and T24 cells infected with pCXbsr/Per similarly expressed periostin protein (Fig. 1A). To quantitatively evaluate the invasive potential of these cell lines, we performed 

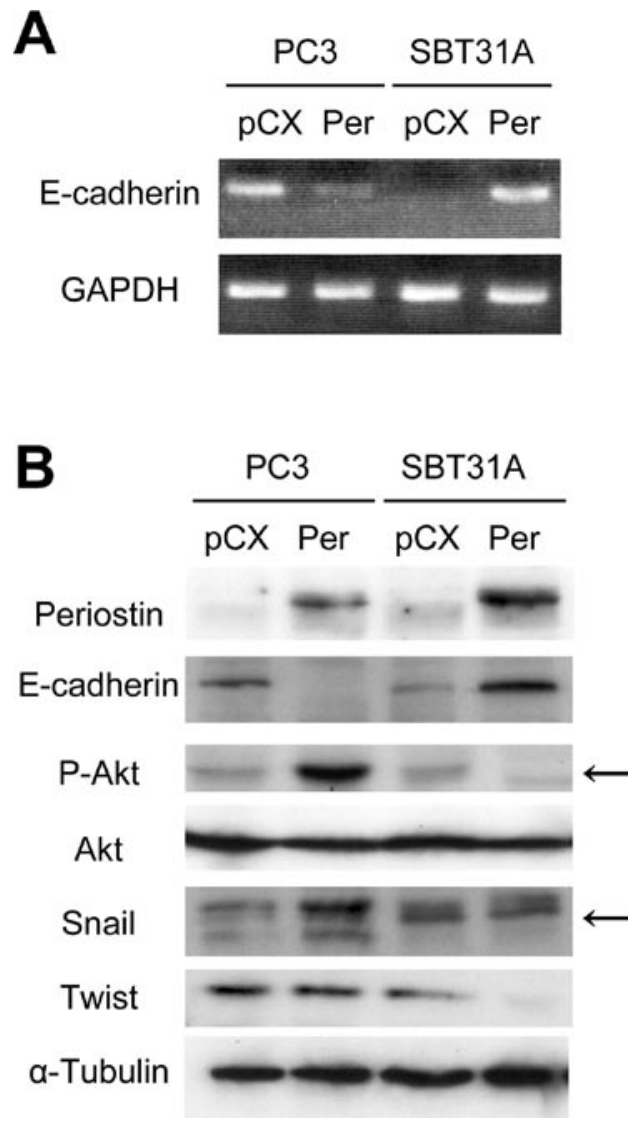

Figure 2. Effects of periostin expression on EMT-related proteins in PC3 and SBT31A cells. (A) RT-PCR analysis of E-cadherin mRNA expression in PC3 and SBT31A cells. The expression of GAPDH was used as an internal control. (B) Alterations of the expression of E-cadherin, Snail, Twist, total Akt (Akt) and phospho Akt (pAkt) by periostin in prostate (PC3) and bladder (SBT31A) cancer cell lines. Cell lysate was separated by SDS-PAGE and immunoblotted for the anti-E-cadherin, anti-periostin, anti-Snail, anti-Twist, anti-phospho Akt (Ser473) and anti-Akt antibodies. The expression of $\alpha$-tubulin was used as an internal control. The arrows show the bands of phosphorylated Akt and Snail.

an in vitro invasion assay using Matrigel Basement Membrane Matrix Invasion Chambers. As shown in Fig. 1B, the in vitro invasiveness of PC3 and DU145 cells expressing exogenous periostin was markedly higher than that of the control cells with vector. On the other hand, the in vitro invasiveness of SBT31A and T24 cells expressing exogenous periostin was markedly lower than that of control cells with vector. These results indicate that periostin has opposite effects on the in vitro invasiveness between prostate and bladder cancer cells.

Opposite regulation of E-cadherin by periostin. To investigate the role of EMT in the opposite regulation of the cell invasiveness by periostin, we examined the effects of periostin expression on the levels of E-cadherin and vimentin in prostate (PC3 and DU145) and bladder (SBT31A and T24) cancer cell lines (Fig. 1A). Periostin downregulated E-cadherin protein expression in PC3 and DU145 cells, but upregulated its expression in SBT31A and T24 cells, indicating that periostin also has opposite effects on the regulation of E-cadherin expression between prostate and bladder cancer cells. However, the levels of vimentin protein were not affected by the expression of exogenous periostin in both
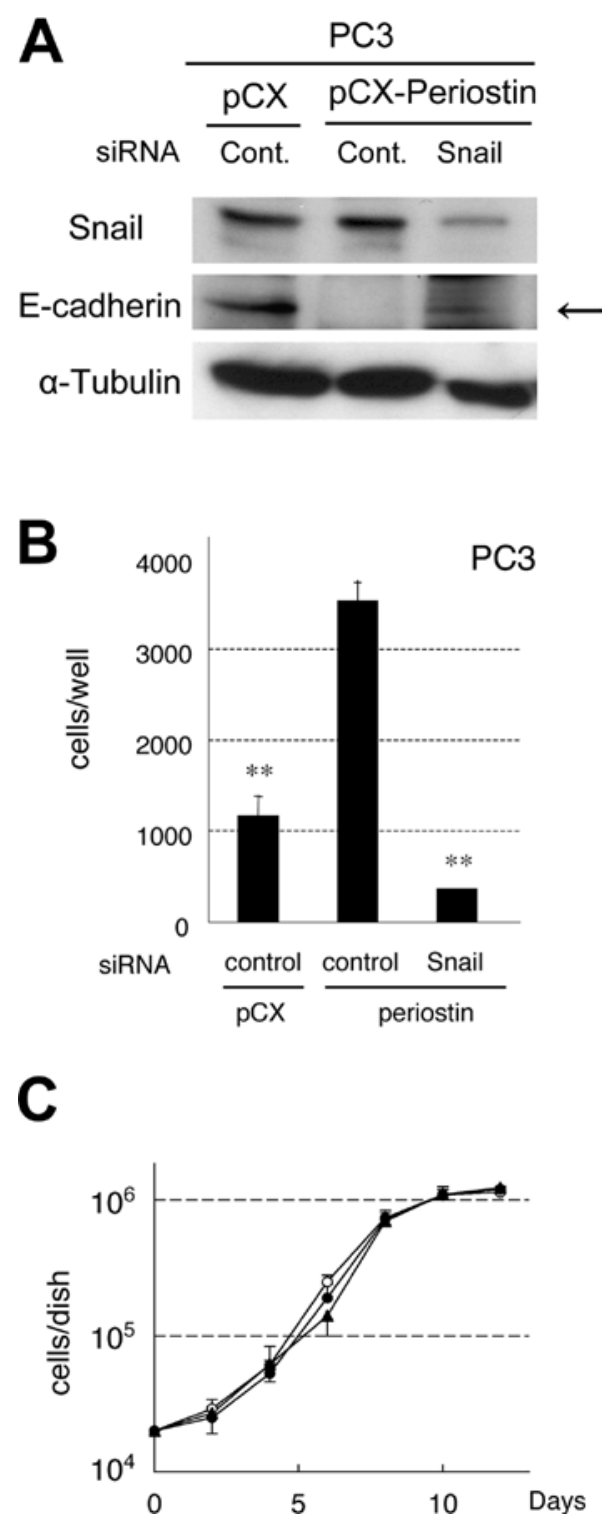

Figure 3. Effect of siRNA targeting human Snail on the enhancement of cell invasiveness by periostin in PC 3 cells. (A) Effects of siRNA targeting Snaill on the expression of endogenous Snail protein in PC3pCX and PC3pCXperiostin cells. These cells were transfected with control siRNA (Cont., 120 pmol) (PC3pCX and PC3pCX-periostin) and with siRNA targeting Snaill (120 pmole) (PC3pCX-periostin) by Lipofectamine 2000 reagent. To confirm the expression of Snail protein in transfected cells, whole lysed extracts were immunoblotted for anti-Snail and anti-E-cadherin antibodies. The expression of $\alpha$-tubulin was used as an internal control. The arrow shows the band of E-cadherin. (B) Effect of siRNA targeting Snaill on the invasiveness of PC3 cells expressing exogenous periostin. The numbers of cells invading through the Matrigel are shown. Each sample was assayed in triplicate. Bars, \pm SD of triplicate chambers for each experiment. ${ }^{* *} \mathrm{p}<0.01$, as compared to the control cells (PC3pCX-periostin, control). (C) Growth curves of PC3pCX and PC3pCX-periostin cells transfected with siRNA. Cells $\left(2 \times 10^{4}\right)$ were seeded in $35-\mathrm{mm}$ culture plates. The growing cells in triplicate plates were trypsinized and counted. Open circle, closed circle, and closed triangle show PC3-pCX cont., PC3-periostin cont., and PC3-periostin siSnail, respectively.

prostate and bladder cancer cell lines. These results indicate that periostin regulates the expression of E-cadherin, a key molecule of EMT, in a cell-type dependent manner, and that this regulation of E-cadherin is inversely correlated with periostin's effect on the invasiveness of prostate and bladder cancer cells. 

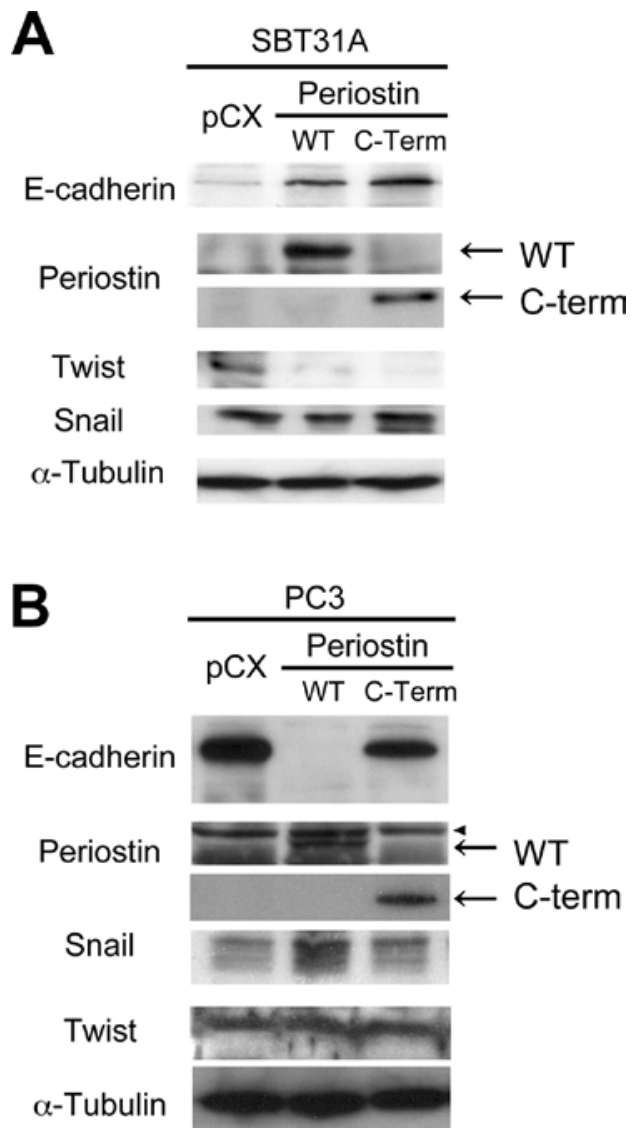

Figure 4. Effects of the C-terminal region of periostin on the expression of E-cadherin, Snail and Twist in PC3 and SBT31A cells. Immunoblot analysis of SBT31A (A) and PC3 (B) cells expressing wild-type (WT) and the C-terminal region (C-term) of periostin. Cell lysate was separated by SDS-PAGE and immunoblotted for anti-E-cadherin, anti-periostin, anti-Snail and anti-Twist antibodies. The expression of $\alpha$-tubulin was used as an internal control. The arrows show the bands of WT and C-term periostin. The arrowhead shows a non-specific band.

To clarify whether or not periostin regulates E-cadherin expression at the transcriptional level, the alterations of E-cadherin mRNA expression by periostin in PC3 and SBT31A cells were examined by RT-PCR. As shown in Fig. 2A, periostin downregulated the mRNA expression of E-cadherin Type I in PC3 cells, whereas in SBT31A cells it upregulated the expression of E-cadherin Type I. The same results were obtained in RT-PCR experiments with E-cadherin Type II primers and in those of DU145 and T24 cells with E-cadherin Type I and Type II primers (data not shown). These results indicate that periostin regulates E-cadherin expression at the transcriptional level. The EMT-related transcription factors Snail and Twist are known to negatively regulate E-cadherin transcription through E-boxes $(17,18)$. To further clarify the mechanism underlying the transcriptional regulation of E-cadherin by periostin, we focused on PC3 and SBT31A cells and examined the effects of periostin on the expression of Snail and Twist in these cancer cells by immunoblot analysis. As shown in Fig. 2B, the upregulation of Snail in PC3 cells and the downregulation of Twist in SBT31A cells were observed. Periostin expression did not affect the expression of Twist in PC3 cells or that of Snail in SBT31A cells. To determine whether or not Snail is required for the enhanced invasiveness by periostin of prostate cancer cells, we examined the effect of siRNA targeting Snail on the invasiveness of PC3 cells expressing periostin. As shown in Fig. 3A, the siRNA targeting Snail had a marked knockdown effect on endogenous expression of Snail in PC3 cells expressing periostin. E-cadherin expression was upregulated by the siRNA for Snail in these cells. The siRNA targeting Snail markedly inhibited the invasiveness of PC3 expressing periostin (Fig. 3B). Cell proliferation was not affected by the expression of the siRNA for Snail (Fig. 3C). These results indicate that Snail contributes to the downregulation of E-cadherin and the enhancement of the invasiveness by periostin in PC3 prostate cancer cells.

Role of the $C$-terminal region of periostin in the regulation of E-cadherin expression in bladder and prostate cancer cells. We previously revealed that the C-terminal region of periostin is sufficient to suppress the in vitro invasiveness of SBT31A bladder cancer cells (12) (Fig. 1B). To clarify whether or not the C-terminal region (C-term) of periostin has the effect on the invasiveness of prostate cancer cells, we performed a Matrigel invasion assay of PC3 cells expressing the C-term mutant. As shown in Fig. 1B, this mutant did not enhance the invasiveness of PC3 prostate cancer cells. Furthermore, we examined the effects of the C-term mutant on the expression of E-cadherin and EMT-related transcription factors, Snail and Twist, in both SBT31A and PC3 cancer cell lines. The $\mathrm{C}$-term mutant of periostin could upregulate E-cadherin and downregulate Twist in SBT31A cells, as well as WT periostin (Fig. 4A). On the other hand, the C-term mutant did not affect the expression of E-cadherin or Snail in PC3 prostate cancer cells, irrespective of the expression of C-term protein (Fig. 4B). These results indicate that the $\mathrm{C}$-terminal region of periostin is sufficient to suppress the cell invasiveness and to increase the expression of E-cadherin via the suppression of Twist in bladder cancer cells, but is not sufficient to enhance invasiveness or to regulate the expression of E-cadherin and Snail in prostate cancer cells. This, in turn, supports the idea that the mechanism underlying the regulation of E-cadherin expression and the invasiveness by periostin differs between bladder and prostate cancer cells.

Effects of periostin on Akt phosphorylation in prostate and bladder cancer cells. Deregulation of the Akt signaling pathway is one of the most common signaling alterations in the development of human malignant cancers (19). Periostin has also been reported to enhance metastatic growth of colon cancer both by preventing stress-induced apoptosis in cancer cells and by augmenting endothelial cell survival via the Akt/ PKB pathway (5). To clarify the role of the Akt signaling pathway in the regulation of invasiveness and E-cadherin expression by periostin in prostate and bladder cancer cells, the alterations of Akt phosphorylation by periostin were examined by immunoblot analysis with phosphorylation-specific antibody for Akt in SBT31A and PC3 cells. As shown in Fig. 2B, exogenous expression of periostin upregulated phosphorylation (Ser473) of Akt in PC 3 cells but downregulated it in SBT31A cells. The expression of Akt protein was not affected by periostin in either PC3 or SBT31A cells. These results also indicate opposite effects of periostin on Akt phosphorylation between prostate and bladder cancer cells. 

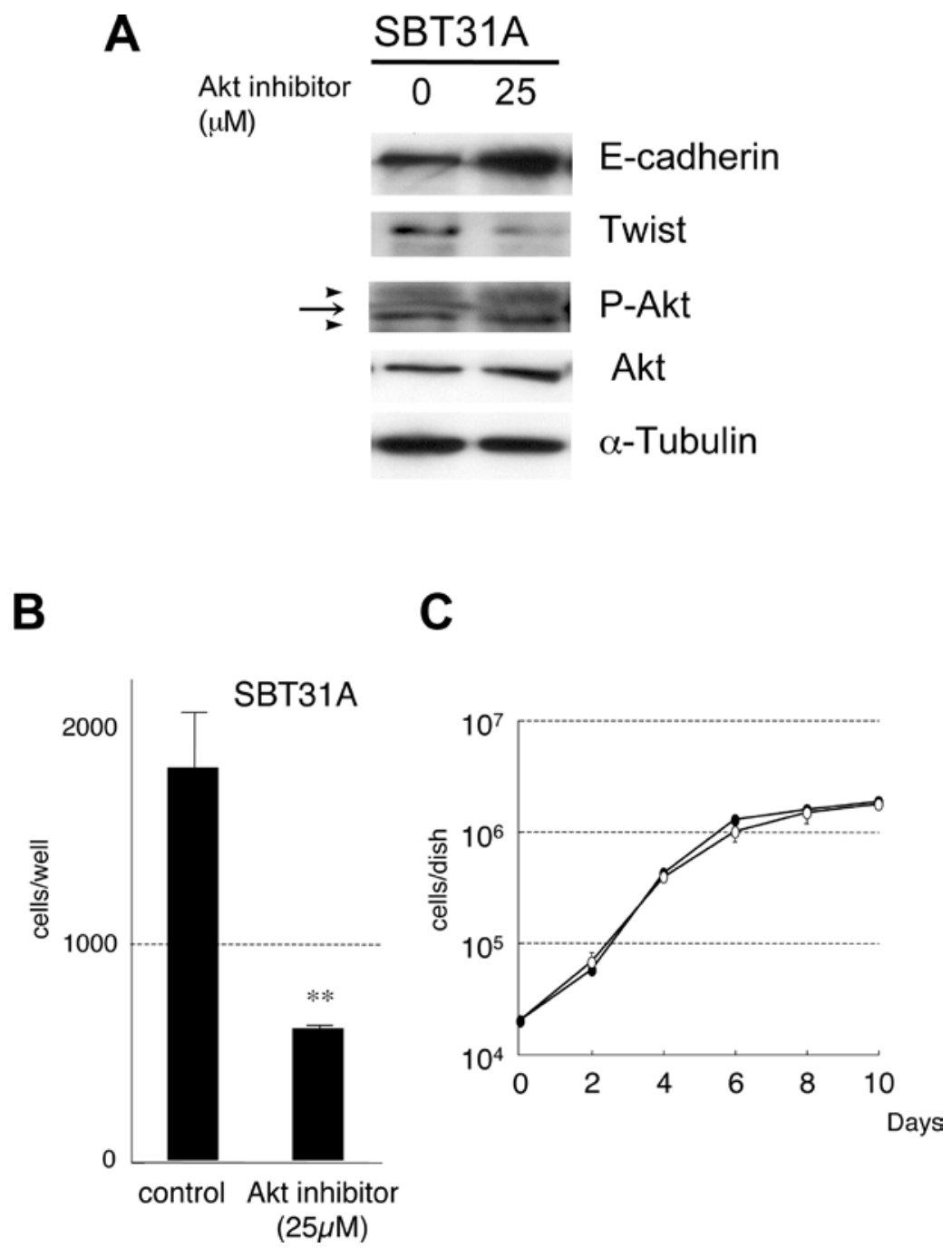

Figure 5. Effects of Akt inhibitor on EMT and invasiveness of SBT31A cells. (A) Immmunoblot analysis of SBT31A cells treated with Akt inhibitor. SBT31A cells were treated with $25 \mu \mathrm{M}$ of Akt inhibitor for $24 \mathrm{~h}$. Cell lysate was separated by SDS-PAGE and immunoblotted for anti-E-cadherin, anti-Twist, antiphospho Akt (Ser473) and anti-Akt antibodies. The expression of $\alpha$-tubulin was used as an internal control. The arrow shows the band of phosphorylated Akt. The arrowheads show non-specific bands. (B) Effect of Akt inhibitor on the invasiveness of SBT31A cells. SBT31A cells were treated with $25 \mu \mathrm{M}$ of Akt inhibitor for $24 \mathrm{~h}$ and inoculated into Matrigel invasion chambers without Akt inhibitor. The numbers of cells invading through the Matrigel are shown. Each sample was assayed in triplicate. Bars, $\pm \mathrm{SD}$ of triplicate chambers for each experiment. ${ }^{* *} \mathrm{p}<0.01$, as compared to the untreated control cells (control). (C) Growth curves of untreated control and Akt-inhibitor-treated SBT31A cells. After treatment with $25 \mu \mathrm{M}$ Akt inhibitor for $24 \mathrm{~h}, 2 \times 10^{4}$ cells were seeded in $35-\mathrm{mm}$ culture plates in the complete culture medium without Akt inhibitor. The growing cells in triplicate plates were trypsinized and counted. Open circle and closed circle represent the control SBT31A cells and SBT31A cells treated with Akt inhibitor $(25 \mu \mathrm{M})$, respectively.

Effects of Akt inhibition on EMT and the invasiveness of bladder cancer cells. To clarify whether or not the inhibition of Akt activity contributes to the upregulation of E-cadherin expression to suppress the invasiveness of bladder cancer cells, we examined the effects of an Akt inhibitor on invasiveness and E-cadherin expression in SBT31A cells. SBT31A cells were treated with $25 \mu \mathrm{M}$ Akt inhibitor for $24 \mathrm{~h}$. This concentration was sufficient to suppress the phosphorylation of Akt without affecting the amount of Akt protein (Fig. 5A). The inhibition of Akt phosphorylation induced the downregulation of Twist and the upregulation of E-cadherin. After the treatment with Akt inhibitor, a Matrigel invasion assay was performed without Akt inhibitor. Treatment with Akt inhibitor significantly suppressed the invasiveness of SBT31A cells (Fig. 5B). After treatment with Akt inhibitor, the proliferation of these cells was also examined in complete culture medium without Akt inhibitor.
The proliferation of SBT31A cells was not affected by Akt inhibitor treatment (Fig. 5C). These results indicate that, like the expression of exogenous periostin, Akt activity is critical to the regulation of both Twist and E-cadherin expression and to the invasiveness of SBT31A cells.

\section{Discussion}

In the present study, we investigated the role of the EMT in the regulation of invasiveness by periostin in human prostate and bladder cancer cell lines and found that periostin regulates E-cadherin expression and cell invasiveness of these cancer cells in a cell-type-dependent manner via Akt phosphorylation and negative regulators of E-cadherin, Snail and Twist (Fig. 6).

EMT is considered an important event during malignant tumor progression, invasion and metastasis $(1,20)$. One of its 


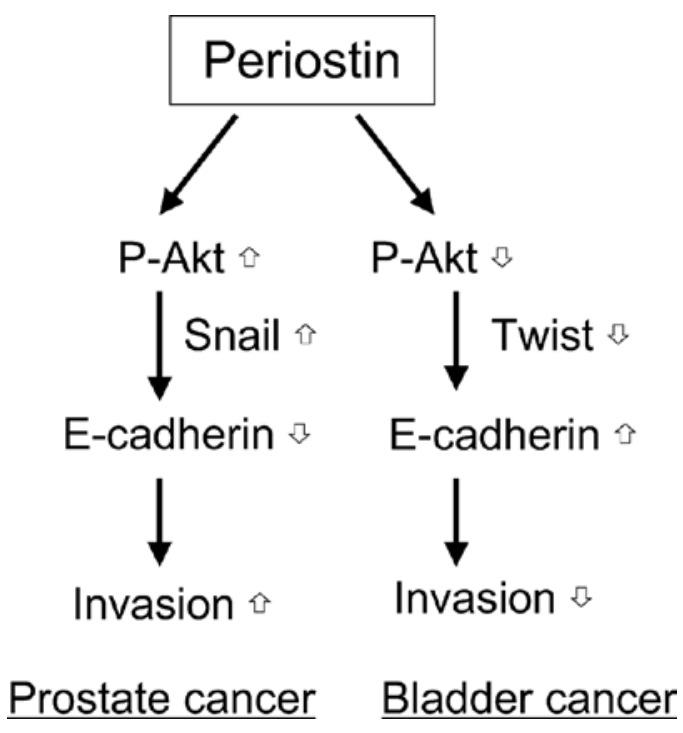

Figure 6. Cell-type-dependent regulation of EMT and cell invasiveness by periostin in prostate and bladder cancer cells.

hallmarks is the loss of E-cadherin expression. Downregulation of E-cadherin has been reported in human bladder and prostate cancer tissues. A reduction or loss of E-cadherin expression was correlated with the progression and patient prognosis of human bladder and prostate cancers $(21,22)$. This change was linked to increased motility and invasive potential. The roles of periostin have also been reported in human bladder and prostate cancer tissues. Epithelial and stromal expression of periostin in normal prostate was detected in 74 and $13 \%$ of tissues examined, respectively (23). Periostin expression increased in the early stages of prostate cancer (Gleason score 6-7) but not in the advanced stages of prostate cancer. Furthermore, stromal expression of periostin was observed in $36 \%$ of Gleason score 6-7 prostate cancers, $65 \%$ of Gleason score 8-10 prostate cancers, and $100 \%$ of bone metastasis tissues. The positive ratio observed for the expression of periostin in tumor-associated stroma was significantly correlated with the degree of malignancy. In contrast, periostin was expressed in $100 \%$ of normal bladder tissues and in $82 \%$ of grade $1,40 \%$ of grade 2 and $33 \%$ of grade 3 bladder cancer tissues, indicating that downregulation of periostin is significantly related to high-grade bladder cancer (12). These findings are consistent with the opposite functions of periostin in the invasiveness between prostate and bladder cancer cell lines.

We previously revealed that the C-terminal region of periostin was sufficient to suppress the invasiveness of human bladder cancer cells and the metastasis of melanoma cells, although both the $\mathrm{N}$-terminal and $\mathrm{C}$-terminal regions are involved in the suppression of these functions of periostin (12). In the present study, we found that the C-terminal region of periostin was also sufficient to induce the upregulation of E-cadherin and the downregulation of Twist in bladder cancer cells. However, it did not affect the regulation of E-cadherin, Snail or invasiveness of prostate cancer cells. These findings also demonstrate that different molecular mechanisms exist for the regulation of EMT and invasiveness between bladder and prostate cancer cells. We showed that the upregulation of
Akt phosphorylation and Snail by periostin is involved in the regulation of E-cadherin and the invasiveness of prostate cancer cells. Julien et al have also shown that Akt upregulates Snail expression via activation of NF- $\kappa \mathrm{B}$ to induce EMT (24). We also found that periostin has opposite effects on Akt phosphorylation between prostate and bladder cancer cells. Ectopic expression of periostin upregulated Akt phosphorylation in prostate cancer cells and downregulated it in bladder cancer cells. Periostin has been reported to promote the metastatic growth of colon cancer by augmenting cell survival via the Akt/PKB pathway (5). Phosphorylated Akt (pAkt) is inversely associated with E-cadherin expression in oral squamous cell carcinoma (25). Akt activation repressed E-cadherin gene transcription by upregulation of the transcription repressors Snail and SIP1, and enhanced the invasiveness of squamous cell carcinoma cells (26). Our results with Akt phosphorylation and the invasiveness of prostate cancer cells are consistent with these previous findings. In the present study, we also showed that both periostin expression and treatment with Akt inhibitor downregulated the Twist transcription factor to enhance the expression of E-cadherin, resulting in the suppression of the invasiveness in bladder cancer cells. Erkan et al have reported that periostin had biphasic effects on the Akt phosphorylation of pancreatic cancer cells (27). They have also shown that periostin significantly decreased Akt phosphorylation at a concentration of $100 \mathrm{ng} / \mathrm{ml}$, whereas it increased Akt phosphorylation at $1 \mu \mathrm{g} / \mathrm{ml}$. Under our experimental conditions, the expression of exogenous periostin was similar between prostate and bladder cancer cells (Fig. 1A), indicating that the concentration of periostin protein in each type of cell was not due to the opposite effect on Akt activity. One or more cell-type-specific factors may contribute to the regulation of Akt phosphorylation by periostin in bladder and prostate cancer cells. Akt, a serine/threonine kinase, is important in regulating key processes involved in tumor growth and progression. Akt phosphorylation was not significantly associated with tumor grade or stage in bladder cancer cells. However, it was found to be significantly higher in non-papillary bladder cancers than in papillary bladder cancers (28). The progression of prostate cancer is accompanied by increased levels of pAkt (29). In the present study, inhibition of Akt activity by periostin or treatment with Akt inhibitor induced the upregulation of E-cadherin and the suppression of invasiveness of bladder cancer cells. These findings suggest that future therapies based on the inhibition of Akt activity may complement conventional treatments by controlling tumor invasiveness and metastasis.

\section{Acknowledgements}

We thank Mrs. Akiyo Ushio for her technical assistance. This project was supported by a Grant-in Aid for Scientific Research (C) (Grant Nos. 19591843 and 17590341 to C.J. Kim and H. Inoue, respectively) from the Ministry of Education, Culture, Sports, Science and Technology of Japan.

\section{References}

1. Thiery JP and Sleeman JP: Complex networks orchestrate epithelial-mesenchymal transitions. Nat Rev Mol Cell Biol 7: 131-142, 2006. 
2. Thiery JP: Epithelial-mesenchymal transitions in tumour progression. Nat Rev Cancer 2: 442-454, 2001.

3. Birchmeier W and Behrens J: Cadherin expression in carcinomas: role in the formation of cell junctions and the prevention of invasiveness. Biochim Biophys Acta 1198: 11-26, 1994.

4. Lindsley A, Snider P, Zhou H, et al: Identification and characterization of a novel Schwann and outflow tract endocardial cushion lineage-restricted periostin enhancer. Dev Biol 307: 340-355, 2007

5. Bao S, Ouyang G, Bai X, et al: Periostin potently promotes metastatic growth of colon cancer by augmenting cell survival via the Akt/PKB pathway. Cancer Cell 5: 329-339, 2004.

6. Shao R, Bao S, Bai X, et al: Acquired expression of periostin by human breast cancers promotes tumor angiogenesis through upregulation of vascular endothelial growth factor 2 expression. Mol Cell Biol 24: 3992-4003, 2004.

7. Sasaki H, Lo KM, Chen LB, et al: Expression of periostin, homologous with an insect cell adhesion molecule, as a prognostic marker in non-small cell lung cancers. Jpn J Cancer Res 92 869-873, 2001.

8. Kudo Y, Ogawa I, Kitajima S, et al: Periostin promotes invasion and anchorage-independent growth in the metastatic process of head and neck cancer. Cancer Res 66: 6928-6935, 2006.

9. Siriwardena BS, Kudo Y, Ogawa I, et al: Periostin is frequently overexpressed and enhances invasion and angiogenesis in ora cancer. Br J Cancer 95: 1396-1403, 2006.

10. Baril P, Gangeswaran R, Mahon PC, et al: Periostin promotes invasiveness and resistance of pancreatic cancer cells to hypoxiainduced cell death: role of the $\beta 4$ integrin and the PI3k pathway. Oncogene 26: 2082-2094, 2007.

11. Soltermann A, Tischler V, Arbogast S, et al: Prognostic significance of epithelial-mesenchymal and mesenchymal-epithelial transition protein expression in non-small cell lung cancer. Clin Cancer Res 14: 7430-7437, 2008

12. Kim CJ, Yoshioka N, Tambe Y, Kushima R, Okada Y and Inoue H: Periostin is down-regulated in high grade human bladder cancers and suppresses in vitro cell invasiveness and in vivo metastasis of cancer cells. Int J Cancer 117: 51-58, 2005.

13. Kim CJ, Isono T, Tambe Y, et al: Role of alternative splicing of periostin in human bladder carcinogenesis. Int J Oncol 32 : 161-169, 2008

14. Yoshioka N, Fuji S, Shimakage M, et al: Suppression of anchorageindependent growth of human cancer cell lines by the TRIF52/ periostin/OSF-2 gene. Exp Cell Res 279: 91-99, 2002.

15. Naviaux RK, Constanzi E, Haas M and Verma IM: The pCL vector system: rapid production of helper-free, high-titer, recombinant retroviruses. J Virol 70: 5701-5705, 1996.

16. Hong L, Sun H, Lv X, Yang D, Zhang J and Shi Y: Expression of periostin in the serum of NSCLC and its function on proliferation and migration of human lung adenocarcinoma cell line (A549) in vitro. Mol Biol Rep 37: 2285-2293, 2010.
17. Giroldi LA, Bringuier PP, de Weijert M, Jansen C, van Bokhoven A and Schalken JA: Role of $\mathrm{E}$ boxes in the repression of E-cadherin expression. Biochem Biophys Res Commun 241: 453-458, 1997.

18. Batlle E, Sancho E, Franci C, et al: The transcription factor snail is a repressor of E-cadherin gene expression in epithelial tumour cells. Nat Cell Biol 2: 84-89, 2000.

19. Cheng GZ, Park S, Shu S, et al: Advances of AKT pathway in human oncogenesis and as a target for anti-cancer drug discovery. Curr Cancer Drug Targets 8: 2-6, 2008.

20. Christiansen JJ and Rajasekaran AK: Reassessing epithelial to mesenchymal transition as a prerequisite for carcinoma invasion and metastasis. Cancer Res 66: 8319-8326, 2006.

21. Rieger-Christ KM, Ng L, Hanley RS, et al: Restoration of plakoglobin expression in bladder carcinoma cell lines suppresses cell migration and tumorigenic potential. Br J Cancer 92: 2153-2159, 2005.

22. Gravdal K, Halvorsen OJ, Haukaas SA and Akslen LA: A switch from $\mathrm{E}$-cadherin to $\mathrm{N}$-cadherin expression indicates epithelial to mesenchymal transition and is of strong and independent importance for the progress of prostate cancer. Clin Cancer Res 13: 7003-7011, 2007.

23. Tsunoda T, Furusato B, Takashima Y, et al: The increased expression of periostin during early stages of prostate cancer and advanced stages of cancer stroma. Prostate 69: 1398-1403, 2009.

24. Julien S, Puig I, Caretti E, et al: Activation of NF- $\kappa$ B by Akt upregulates Snail expression and induces epithelium mesenchymal transition. Oncogene 26: 7445-7456, 2007.

25. Lim J, Kim JH, Paeng JY, et al: Prognostic value of activated Akt expression in oral squamous cell carcinoma. J Clin Pathol 58: 1199-1205, 2005.

26. Grille SJ, Bellacosa A, Upson J, et al: The protein kinase Akt induces epithelial mesenchymal transition and promotes enhanced motility and invasiveness of squamous cell carcinoma lines. Cancer Res 63: 2172-2178, 2003.

27. Erkan M, Kleeff J, Gorbachevski A, et al: Periostin creates a tumor-supportive microenvironment in the pancreas by sustaining fibrogenic stellate cell activity. Gastroenterology 132: 1447-1464, 2007.

28. Harris LD, De La Cerda J, Tuziak T, et al: Analysis of the expression of biomarkers in urinary bladder cancer using a tissue microarray. Mol Carcinog 47: 678-685, 2008

29. Malik SN, Brattain M, Ghosh PM, et al: Immunohistochemical demonstration of phospho-Akt in high Gleason grade prostate cancer. Clin Cancer Res 8: 1168-1171, 2002. 